\title{
Comprensión de textos expositivos académicos en e-book Reader y en papel: influencia del conocimiento previo de dominio y la aptitud verbal
}

\section{Comprehension of academic expository texts in e-book Reader vs. paper: influence of prior domain knowledge and verbal aptitude}

\author{
Silvia Piovano ${ }^{1}$ \\ Natalia Irrazabal ${ }^{24}$ \\ Débora I. Burin ${ }^{34}$ \\ ${ }^{1}$ Departamento de Psicología y Ciencias Pedagógicas, Universidad CAECE. Argentina \\ ${ }^{2}$ Facultad de Ciencias Sociales, Universidad de Palermo. Argentina \\ ${ }^{3}$ Facultad de Psicología, Universidad de Buenos Aires. Argentina \\ ${ }^{4}$ Consejo Nacional de Investigaciones Científicas y Técnicas. Argentina
}

\begin{abstract}
Resumen: En un estudio del que participaron 102 estudiantes universitarios, se comparó la comprensión en la lectura de textos expositivos académicos impresos versus en formato de $e$-book Reader. Se examinaron los efectos del soporte, del conocimiento específico de dominio previo del lector y la aptitud verbal. Asimismo se indagaron las actitudes de los estudiantes hacia los soportes textuales antes y después de la experiencia. Los resultados sugieren que el e-book Reader beneficiaría a los lectores competentes, y/o que podría promover mayor actividad metacognitiva en los procesos de comprensión. Asimismo, las opiniones hacia el e-book Reader cambiaron después de la experiencia, aunque los juicios de autoevaluación evidenciaron que se sigue prefiriendo el formato impreso.
\end{abstract}

Palabras clave: E-book Reader, comprensión lectora, textos expositivos, aptitud verbal, metacognición

Abstract: In a study involving 102 university students, reading comprehension of printed academic texts versus e-book Reader format was compared. Effects of specific previous domain knowledge and verbal ability were examined. Students' attitudes towards the e-book Reader before and after the experiment were also investigated. Results suggest that the e-book Reader would benefit more competent readers, and that it could promote greater metacognitive activity in comprehension processes. Also, attitudes towards the e-book Readerchanged after the experience, although selfevaluation judgments showed that the printed format is still preferred.

Keywords: E-book Reader, reading comprehension, expository texts, verbal aptitude, metacognition

Cómo citar este artículo:

Piovano, S., Irrazabal, N., \& Burin, D. I. (2018). Comprensión de textos expositivos académicos en e-book Reader y en papel: influencia del conocimiento previo de dominio y la aptitud verbal. Ciencias Psicológicas, 12(2), 177-185. doi: https://doi.org/10.22235/cp.v12i2.1680.

Correspondencia: Silvia Piovano. Universidad CAECE, Argentina. Mario Bravo 1259 (1175). CABA; e-mail: sipiovano@gmail.com. Natalia Irrazabal. Universidad de Palermo - CONICET, Argentina; e-mail: nirrazabal@psi.uba.ar. Débora I. Burin. Universidad de Buenos Aires - CONICET, Argentina; e-mail: dburin@psi.uba.ar 
La comprensión de textos expositivos académicos es una de las habilidades que se requieren para el logro exitoso de los estudios universitarios. Leer consiste en llevar a cabo procesos mentales para construir representaciones del significado del texto. El lector utiliza información explícita y realiza inferencias para construir e integrar una representación semántica, cohesiva y coherente del significado local y global del texto escrito (Kintsch, 1996; León \& Escudero, 2003). El modelo de van Dijk y Kintsch (1983) postula que el significado se construye en dos niveles, la Base de Texto y el Modelo de Situación. La Base de Texto es una construcción que incluye aspectos superficiales (identificación y reconocimiento de palabras y las relaciones sintácticas y semánticas entre ellas) y proposicionales (construcción de las relaciones lógicas en los enunciados). El Modelo de Situación está más alejado de lo literal y se forma con representaciones semánticas integradas en la memoria de largo plazo. Además del dominio de habilidades lingüísticas, que posibilita la coherencia y cohesión, el texto expositivo requiere conocimiento previo acerca de la temática a la que se refiere. Por lo general, los textos académicos son fuente de información compleja y requieren de un lector ya iniciado o experto (Graesser, McNamara, \& VanLehn, 2005; León \& Escudero, 2003).

Entre los factoresque llevan a diferencias individuales en comprensión se destacan el conocimiento previo, la aptitud verbal y las actividades metacognitivas (Graesser et al., 2005; Irrazabal, Burin, \& Saux, 2012; McNamara, Kintsch, Songer, \& Kintsch, 1996; Perfetti, 2007). El nivel de conocimiento previo alude al conocimiento almacenado que pueda poseer el lector, tanto dela temática específica, como de la superestructura textual. La aptitud verbal representa la capacidad de un sujeto para comprender palabras y sus relaciones sintácticas y semánticas (Cortada de Kohan, 2004). Intervienen el vocabulario, la identificación de conceptos y su utilización, lo que posibilita la construcción del significado a través de procesos de activación e inhibición a medida que se lee un texto (Cortada de Kohan, 2004; Perfetti, 2007; Perfetti, \& Stafura, 2014). La metacognición abarca diferentes aspectos de auto-regulación de la conducta, como la planificación, el monitoreo, y actividades estratégicas como subrayado, resúmenes o auto-explicaciones (Afflerbach, Pearson, \& Paris, 2007).
Con la aparición de las tecnologías digitales, las investigaciones han hallado diferencias entre la lectura a través de textos impresos y la que se realiza en entornos digitales. En general se plantea que el primer soporte sería facilitador de una lectura reflexiva y profunda, mientras que la lectura en pantalla de computadora es vista como superficial, rápida y promotora de desorientación (Albarello, 2011; Amadieu, van Gog, Paas, \& Mariné, 2009; Burin, Kahan, Irrazabal, \& Saux, 2010, 2014). A fines del siglo XX el ebook-Reader o libro digital plantea una nueva manera de leer como solución a las dificultades que se han advertido en los otros medios digitales: lectura similar al libro en papel, retroalimentación de la pantalla, omnipresencia (Cordón García, Alonso Arévalo, \& Martín Rodero, 2010; Rich, 2012). El eBook es el libro en formato digital y el eReader es un dispositivo de lectura. Por medio de este dispositivo se busca asimilar la lectura en un soporte electrónico a la lectura en papel (Benéitez, Camarero, \& San José, 2012; Wexelbaum, Miltenoff, \& Parault, 2011). La consideración de este supuesto teórico genera así la necesidad de investigar si la lectura realizada a través del eReader (en adelante, "Reader") promueve una lectura comprensiva y exitosa en comparación con la realizada en el libro impreso.

Se pueden apreciar dos grupos de investigaciones sobre la lectura en nuevos soportes digitales, en particular el Reader, las orientadas a actitudes u opiniones y las referidas a comprensión lectora de textos.

Los estudios que se centran en las opiniones y actitudes sobre los soportes de lectura y su utilización (Ahlroos \& Hahto, 2012; Baker, 2010; Connell, Bayliss, \& Farmer, 2012; David \& Woody, 2013; Lartigue, Rutledge, \& Rice, 2013; Margolin, Driscoll, Toland, \& Kegler, 2013; Rockinson-Szapkiw, Courduff, Carter, \& Bennett, 2012; Rockinson-Szapkiw, Wendt, \& Lunde, 2013; Zhang, 2012). Sostienen, de modo general, que el Reader constituye un avance en términos de portabilidad, facilidad de uso, precio y accesibilidad. Aunque no es muy extendido su uso cuando se toman muestras grandes como en Alonso-Arévalo, Cordón-García y Gómez-Díaz (2011); Rubio y Giménez (2014); Zhang (2012). En general las personas que ya poseen un Reader afirman que la lectura no es radicalmente diferente del papel impreso (Alonso Arévalo et al., 2011; Fernández Gómez, Cordón García, Alonso Arévalo, \& Gómez Díaz, 2013; Gielen, 2010; Ra- 
inie, Zickuhr, Purcell, Madden, \& Brenner, 2012; Zhang, 2012). La falta de conocimiento hace que se reconozcan problemas. No obstante, una vez que las personas lo usan, cambian las actitudes hacia el dispositivo, pero continúa la preferencia por el texto impreso para estudiar (Baker, 2010; Connell et al., 2012). Algunos encuentran objetable que no se puedan hacer anotaciones (en las versiones de Reader como el Kindle 3 y el Sony ya se incorporó esta posibilidad), que no tenga enlaces, o que no se puedan realizar otras actividades (Campbell, Callaghan, McGarvie, \& Hynd, 2013; Connell et al., 2012; Lartigue et al., 2013; Rubio \& Giménez, 2014; Shurtz \& von Isenburg, 2011). Algunas investigaciones reconocen mayor rapidez en la lectura en Reader como Lartigue et al. (2013) pero otras, plantean lo contrario porque la lectura en el dispositivo les requiere mayor tiempo de lectura o utilizanmás estrategias que las del texto impreso (Connell et al., 2012; Davis \& Woody, 2013; Shibata \& Omura, 2012).

En investigaciones con muestras más grandes (Alonso Arévalo et al., 2011; Federación de Gremios de Editores y Ministerio de Cultura Español, 2011; Rainie et al., 2012; Zhang, 2012) se halla que el uso de un Reader se asocia a mayor ingreso, mayor educación y una edad menor a 50 años.

Las investigaciones de Baker (2010), Connell et al. (2012), Margolin et al. (2013), Schugar, Schugar y Penny (2011), Rockinson-Szapkiw et al. (2012) y Rockinson-Szapkiw et al. (2013) incluyen una manipulación experimental de la lectura en distintos soportes (impreso, Reader, tablet), medidas estandarizadas de rendimiento lector y de actitudes hacia la lectura y la tecnología, un número mayor de participantes y un tratamiento estadístico inferencial. En esos estudios se llevan a cabo mediciones del grado de comprensión a partir de la lectura de textos narrativos o expositivos con diferentes soportes. Por ejemplo, puntuaciones en los resultados de tareas en impreso y Reader en Rockinson-Szapkiw et al. (2012); comparación de los niveles de comprensión lectora evaluados con el QAR (NookReader e impreso) en Schugar et al.(2011); comprensión textual de opción múltiple en Kindle 4, iPadde Apple y material impreso (Connell et al., 2012).

A partir de las mediciones del grado de aprendizaje, en general no se encontraron diferencias significativas en el rendimiento lector en función de los soportes (Baker, 2010; Connell et al., 2012; Margolin et al., 2013; Rockinson-Szapkiw et al.,
2012, 2013; Schugar et al., 2011).Se hallaron diferencias en la autopercepción del rendimiento, las cuales a su vez estaban relacionadas con las afinidades hacia la lectura o la tecnología (Baker, 2010). Rockinson-Szapkiw et al. (2013) reconocieron diferencias en el aprendizaje que pudieron realizar los estudiantes en los dispositivos Reader, pues se mostraron más dispuestos a utilizar estrategias cognitivas y de autorregulación. Margolin et al. (2013) no encontraron diferencias en la lectura de textos narrativos y expositivos en función del soporte (impreso, Reader, pantalla de PC). Connell et al. (2012) midieron tiempos de lectura más rápidos en impreso, que era considerado mejor para estudiar, por su parte, la tablet y el Reader eran elegidos para lecturas recreativas (aunque el iPad podría seleccionarse para estudiar, en Connell et al., 2012). En el estudio realizado por Schugar et al. (2011) se compararon las habilidades de lectura específicamente a través de la utilización de estrategias de monitoreo relacionadas con la comprensión. Demuestran de esta forma, que las actividades estratégicas que los estudiantes realizan para comprender textos impresos no se realizan en forma automática, lo que equivale a decir que no son transferidas al dispositivo Reader de manera inmediata.

En síntesis estas investigaciones (Ahlroos \& Hahto, 2012; Baker, 2010, Campbell et al., 2013; Connell et al., 2012; Margolin et al., 2013; Nielsen, 2010; Rockinson-Szapkiw et al., 2012; Schugar et al., 2011; Zhang, 2012) sugieren similar rendimiento lector en formato impreso y Reader o mejor para impreso, se reconocen dificultades en el uso por falta de conocimiento del dispositivo y centran la investigación sobre el proceso lector en condiciones de aprendizaje.

La presente investigación indaga no sólo el efecto del soporte y las actitudes hacia el Reader después de su utilización, sino también el efecto de factores de conocimiento previo y la aptitud verbal, típicas en el estudio de comprensión lectora. El objetivo general del estudio fue comparar la comprensión en la lectura de textos expositivos académicos impresos, con las realizadas en Reader. Se consideró como hipótesis general que existían diferencias entre el rendimiento comprensivo en la lectura de textos expositivos académicos en soporte impreso y en Reader, considerando también el conocimiento previo específico de dominio y el nivel de aptitud verbalde los estudiantes universitarios. Se analizaron los efectos de los 
factores y sus interacciones en el rendimiento en comprensión y también los efectos del soporte en la auto-evaluación del rendimiento.

\section{Método}

\section{Participantes}

Participaron 134 estudiantes de las Licenciaturas de Psicología, de Psicopedagogía de Psicomotricidad, de Gestión Educativa y de Educación de una universidad privada en la Ciudad de Buenos Aires. Se realizó una prueba piloto con 32 estudiantes (edad $M=28,07, S D=4,17)$, en el cual se probaron los materiales y el procedimiento, y el estudio propiamente dicho con 102 estudiantes $(M=31,1, S D=7,9)$. Su participación fue voluntaria y todos prestaron consentimiento informado por escrito.

Los criterios de inclusión fueron: ser estudiantes universitarios entre 20 y 50 años, de los últimos años $\left(4^{\circ}\right.$ o $\left.5^{\circ}\right)$ de las carreras antes mencionadas, en las que se dictan materias con contenidos de aprendizaje, cognición, atención y lenguaje. Se consideraron como criterios de exclusión: edad menor o mayor a la establecida, puntaje en el rango de déficit en la prueba de Aptitud Verbal.

\section{Materiales}

Para llevar a cabo el estudio, se utilizaron los siguientes materiales:

- Cuestionario sobre datos demográficos y experiencia de lectura en textos impresos y digital. El cuestionario preguntaba edad, sexo, carrera, tipo de texto que lee y para qué, soportes en los que lee (impresos, digitales), posesión de un dispositivo Ipad y/o Reader, explicación acerca de la no posesión o posesión de un Reader. Con este cuestionario se midió la posesión previa de dispositivos digitales de lectura, específicamente la utilización de Reader.

- Baires-A. Test de Aptitud Verbal versión abreviada (Cortada de Kohan, 2004). Este test, de administración colectiva, indaga la aptitud verbal a través de dos subtests de opción múltiple: sinónimos y definiciones. Con el presente test se operativizó la aptitud verbal entendida como el total de respuestas correctas.

- Textos expositivos (Burin et al., 2014, Burin, Barreyro, Saux, \& Irrazabal, 2015). Se emplearon cuatro textos expositivos, dos sobre psicología cognitiva (Memoria, Lenguaje) y dos sobre ciencias naturales (Física, Astronomía). Dados los criterios de inclusión de la muestra en estudio, los dos primeros se consideraron textos de Alto conocimiento previo y los dos segundos de Bajo conocimiento previo. Los cuatro tienen similar longitud (entre 710 y 720 palabras) y estructura argumentativa: un concepto general, dos conceptos subordinados, desarrollo y ejemplos, y una conclusión o tema que los vincula al final.

Los textos fueron implementados en dos versiones: impreso en papel, y en un Reader modelo Sony PRS-T2. En ambos casos el tamaño de letra, la cantidad de páginas y división del texto fue la misma.

- Cuestionario de comprensión de textos expositivos. Cada texto fue seguido de una prueba de comprensión que tenía preguntas literales o de inferencias puente (10 preguntas de respuesta V/F. Ejemplo: "La memoria de corto almacena información por varias horas"; "Se ha comprobado la existencia de una sola fuerza sub-atómica.") y preguntas sobre inferencias elaborativas (2 preguntas múltiple-choice. Ejemplo: "Indique cuál de las siguientes frases representa mejor el tema que ha leído..."). La cantidad de respuestas correctas a las preguntas literales y de inferencias puentes medían el nivel de comprensión a nivel de Base de Texto, por su parte la cantidad de respuestas correctas en las preguntas acerca de las inferencias elaborativas medían la comprensión a nivel del Modelo de Situación.

- Cuestionario de Autoevaluación. Una vez que habían realizado las tareas, se preguntaba por su autoevaluación respecto del soporte en el que pudo comprender mejor los textos expositivos, en preguntas cerradas con opciones de respuesta. También se preguntaba sobre la opinión después de la experiencia de uso del Reader en preguntas cerradas tipo Likert. Se utilizó el mismo para describir los juicios de las personas respecto de su propio rendimiento.

\section{Procedimiento}

Previo al estudio propiamente dicho, se realizó un estudio piloto $(N=32)$ en el cual se pusieron a prueba los materiales y ajustó el procedimiento general.

Se convocó a los alumnos a su posible participación, explicando el propósito general del estu- 
dio. Aquellos que aceptaban se reunían en sesiones de testeo colectivo (en grupos de 30 personas) de aproximadamente media hora donde se firmaba el consentimiento informado y se administraba el Test Baires-A de Aptitud Verbal. Luego, cada uno participaba de una entrevista individual en la que realizaron las tareas de lectura y comprensión y los cuestionarios, que duraba entre cuarenta minutos y una hora.

Cada uno leía cuatro textos, dos de alto y dos de bajo conocimiento previo. A su vez variaba el soporte en que los leían, dos impresos y dos en Reader, en orden contrabalanceado. Después de la lectura de cada texto respondían el cuestionario de comprensión correspondiente. Al terminar los textos respondían el Cuestionario de Autoevaluación acerca de su autopercepción del rendimiento lector en cada uno y su valoración sobre el dispositivo Reader.

\section{Diseño}

Se llevó a cabo un estudio cuasi-experimental. Se variaron sistemáticamente dos factores intrasujeto (Soporte y Conocimiento Previo), y se evaluó una variable de sujeto (Aptitud Verbal). Se tomaron como variables dependientes: cantidad de aciertos en cuestionarios de Comprensión en Base de Texto y en Modelo de Situación. Además se incluyeron medidas descriptivas de autoevaluación de la lectura en distintos Soportes (Impreso y Reader) y actitudes hacia el e-bookReader.

\section{Resultados}

Se realizaron análisis de varianza mixtos con los factores Conocimiento Previo, Soporte y Aptitud Verbal, sobre Comprensión a nivel de Base de Texto y a nivel de Modelo de Situación, ambas consideradas como porcentaje de respuestas correctas.

La Aptitud Verbal se dividió en cuartiles en función de los puntajes percentilares en el BairesA Total. Luego se seleccionaron el primer $(n=$ $28)$ y último cuartil $(n=26)$ para considerar sus interacciones con los otros factores.

Luego, se analizaron los resultados sobre la Comprensión a nivel de Base de Texto (ver descriptivos en tabla 1, expresados en porcentaje). Se realizó un ANOVA mixto con los factores intrasujetos de Conocimiento Previo (Alto, Bajo) y Soporte (Papel, Reader) y el factor inter-sujetos de aptitud verbal (Alta, Baja). El efecto del Conocimiento Previo no fue significativo, $F(1,52)=$ $1.72, p=.195$, eta cuadrado $=0.032$, tampoco fue significativo el efecto del Soporte, $F(1,52)=3.80$, $p=.057$, eta cuadrado $=0.068$, ni de la Aptitud, $F$ $(1,52)=1.49, p=.227$, eta cuadrado $=0.028$. Por su parte, resultó significativa la interacción entre el soporte y la aptitud verbal, $F(1,52)=5.99, p$ $=.018$, eta cuadrado $=0.103$. Análisis pareados posteriores revelaron que para los participantes de baja aptitud verbal no hay diferencias entre las medias según el soporte (impreso: $M=71.78$, $S D=1.59$; Reader: $M=70.89, S D=14.20)$, pero para los participantes de alta aptitud verbal, el rendimiento fue mejor con el Reader $(M=77.88$, $S D=20.08)$ que con el texto impreso $(M=70.35$, $S D=14.35)$.

Tabla 1

Estadísticos descriptivos en comprensión a nivel de Base de Texto en función del Conocimiento Previo, Soporte y grupo de Aptitud Verbal

\begin{tabular}{lllll}
\hline & $\begin{array}{c}\text { Aptitud } \\
\text { Verbal }\end{array}$ & Media & SD & $N$ \\
\hline Bajo & Baja & 71.07 & 16.40 & 28 \\
$\begin{array}{l}\text { Conocimiento } \\
\text { Impreso }\end{array}$ & Alta & 68.85 & 12.10 & 26 \\
& Total & 70.00 & 14.40 & 54 \\
Bajo & Baja & 70.00 & 14.14 & 28 \\
Conocimiento & Alta & 75.00 & 15.03 & 26 \\
Reader & Total & 72.41 & 14.65 & 54 \\
& Baja & 72.50 & 15.54 & 28 \\
Alto & Alta & 71.15 & 16.57 & 26 \\
Conocimiento & Total & 71.85 & 15.90 & 54 \\
Impreso & Baja & 71.79 & 14.41 & 28 \\
& Alta & 80.77 & 13.24 & 26 \\
Alto & Total & 76.11 & 14.46 & 54 \\
Conocimiento & & & & \\
Reader & & & & \\
\hline
\end{tabular}

De manera similar se analizó el rendimiento respecto del Modelo de Situación. La tabla 2 muestra los estadísticos descriptivos para la comprensión (porcentaje de respuestas correctas) en función del Conocimiento Previo, la Aptitud Verbal y el Soporte. El efecto del Conocimiento Previo no fue significativo, $F(1,52)=3.55, p=$ .065 , eta cuadrado $=0.064$, tampoco resultó significativo el efecto del Soporte, $F(1,52)=0.00, p$ $=.961$, eta cuadrado $=0.000$, por su parte, sí fue significativa la Aptitud Verbal, $F(1,52)=6.95 p$ $=.011$, eta cuadrado $=0.118$ ) así como la interacción entre el Conocimiento Previo y el Soporte, $F(1,52)=6.56, p=.013$, eta cuadrado $=0.112)$. 
Los participantes de alta aptitud verbal comprenden mejor que los de baja aptitud verbal (alta: $M$ $=55.28, S D=31.02$ versus baja: $M=42.63, S D$ $=29.51)$. En cuanto a la interacción entre Conocimiento Previo y Soporte, análisis pareados posteriores mostraron que para los textos de alto conocimiento previo no hubo diferencias en el rendimiento; pero para los de bajo conocimiento previo, hubo mayor cantidad de respuestas correctas con el Reader $(M=50.30, S D=32.58)$ que con el texto impreso $(M=39.81, S D=30.53)$.

Tabla 2

Estadísticos descriptivos en comprensión a nivel de Modelo de Situación en función Conocimiento Previo, Soporte y grupo de Aptitud Verbal

\begin{tabular}{lcccc}
\hline & $\begin{array}{c}\text { Aptitud } \\
\text { Verbal }\end{array}$ & Media & SD & $N$ \\
\hline \multirow{3}{*}{ Bajo Conocimiento } & Baja & 29.46 & 27.26 & 28 \\
Impreso & Alta & 50.96 & 30.39 & 26 \\
& Total & 39.81 & 30.52 & 54 \\
Bajo Conocimiento & Baja & 41.96 & 31.95 & 28 \\
Reader & Alta & 58.65 & 31.57 & 26 \\
& Total & 50.00 & 32.57 & 54 \\
Alto Conocimiento & Baja & 54.46 & 30.46 & 28 \\
Impreso & Alta & 60.57 & 32.53 & 26 \\
& Total & 57.40 & 31.33 & 54 \\
Alto Conocimiento & Baja & 44.64 & 28.34 & 28 \\
Reader & Alta & 50.96 & 29.56 & 26 \\
& Total & 47.68 & 28.84 & 54 \\
\hline
\end{tabular}

En relación a la Autoevaluación del rendimiento lector de los textos expositivos, se analizó qué soporte llevó a una mejor comprensión según la percepción de los participantes. La tabla 3 muestra la percepción de los participantes frente a la pregunta directa: "Pude comprender mejor el texto que leí en Reader / Impreso / Ambos". La mayoría de los participantes, aproximadamente la mitad del total $(56.90 \%)$, considera que ambos soportes tuvieron resultados equivalentes. En segundo lugar, con aproximadamente un 30\% de adhesión, resultó la opción "impreso". La respuesta "Reader" se ubica última y con un bajo porcentaje (aproximadamente 10\%).

Del total de la muestra, solo el $4.90 \%$ ( 5 personas) poseían Reader, y un $16.70 \%$ una tablet. El $95 \%$ que no tenía Reader afirmó al comienzo del estudio que no deseaba poseerlo porque no les interesaba o porque no se sentían cómodos.
Al finalizar la tarea experimental, la opinión de los participantes varió en forma favorable por la experiencia de uso. Se consideró mayoritariamente (40\%) un complemento del texto impreso y en forma similar, que no era ni mejor ni peor que leer en impreso $(22 \%)$ o era mejor que leer en pantalla (21\%). Minoritariamente consideraron al Reader como peor que leer un libro impreso $(9 \%)$ o un complemento de la pantalla (4\%), mejor que el impreso $(2 \%)$, peor leer en pantalla $(1 \%)$ o no sabían qué responder $(1 \%)$.

Tabla 3

Percepción del Rendimiento Lector

\begin{tabular}{lcc}
\hline & Frecuencia & Porcentaje \\
\hline Reader & 12 & 11.80 \\
Impreso & 32 & 31.40 \\
En los dos & 58 & 56.90 \\
Total & 102 & 100.00 \\
\hline
\end{tabular}

\section{Discusión}

Este estudio se ha centrado en la comprensión lectora de textos expositivos en el dispositivo Reader. Se ha evaluado el impacto del soporte en relación al impreso y sus interacciones con el conocimiento previo que se posee sobre el tema y la aptitud verbal. De esta forma, se comparó la comprensión en la lectura de textos expositivos académicos impresos con la realizada en Reader, a través de tareas de lectura de textos con contenido científico, de alto o bajo conocimiento previo específico de dominio para una muestra de estudiantes universitarios, y tomando en cuenta el nivel de aptitud verbal.

El soporte (impreso vs. Reader) en sí no marcó ninguna diferencia en relación a la comprensión lectora. Si no que su efecto dependió de la aptitud verbal y del conocimiento previo, ya que se encontraron interacciones con la aptitud verbal a nivel de Base de Texto y con el conocimiento previo a nivel de Modelo de Situación. Por lo tanto, en principio, se puede afirmar que el soporte sí produciría diferencias en la comprensión, de igual modo que Schugar et al. (2011) y Davis y Woody (2013), pero en interacción con las variables de sujeto. La ausencia de diferencias entre impreso y Reader hallada por Baker (2010), Connell et al. (2012), Margolin et al. (2013) y Rockinson-Szapkiw et 
al. (2013) podría deberse a que no consideraron características de los lectores, como las incluidas en el presente estudio.

Respecto de la aptitud verbal (considerando los grupos extremos dentro de una muestra universitaria), como era esperable tuvo efectos significativos por sí sola, en la comprensión (Cortada de Kohan, 2004; Graesser et al., 2005). Además, específicamente en cuanto su relación con el soporte, los lectores de alta aptitud verbal armaron una Base de Texto más completa con el Reader que con el texto impreso. Esto significaría que los lectores con alta aptitud verbal pueden lograr un mejor rendimiento lector con un dispositivo más novedoso.

En cuanto a la relación entre soporte y conocimiento previo, para la comprensión a nivel de Modelo de Situación, hubo una interacción entre ambos factores. Cuando los lectores tenían alto conocimiento previo sobre el texto, el rendimiento era similar en ambos soportes. En cambio, cuando los lectores no disponían de mucho conocimiento sobre el tópico del texto, se veían beneficiados por el soporte digital. Este hallazgo explicaría los resultados de Connell et al. (2012), cuyos participantes poseían un alto conocimiento previo sobre el tema a leer y no encontraron diferencias entre impreso y Reader. ¿Cómo entender entonces que un dispositivo novedoso beneficia la comprensión en textos sobre temas poco conocidos? Esto se hallaría en línea con Davis y Woody (2013), Margolin et al. (2013) y Rockinson-Szapkiw et al. (2013) quienes hallaron que los lectores utilizaban más actividades metacognitivas en los dispositivos Reader, ya que el nuevo dispositivo les planteaba desafíos a los que debían responder para lograr una comprensión exitosa. En este sentido, se podría pensar que en los textos de mayor conocimiento previo era más fácil derivar una idea integrada del contenido; en cambio, en los de menor conocimiento previo, la mayor actividad metacognitiva promovida por el Reader podría haber contribuido al procesamiento de las ideas centrales. Futuras investigaciones podrían ahondar en el papel de la regulación metacognitiva de la lectura con las nuevas tecnologías.

Respecto de los aspectos metacognitivos analizados en el presente estudio, se analizó la autoevaluación del rendimiento lector de los textos expositivos en función del soporte. Aunque los datos son descriptivos de esta muestra, sugieren que, luego de hacer la experiencia con los textos en ambos formatos, la mitad de los participantes los evalúa de forma similar; y que en la otra la otra mitad, que se inclina por uno u otro, el formato impreso se lleva la preferencia. Es decir que los participantes consideraron que su lectura había sido mejor en los textos en papel. Estos resultados serían similares a Ahlroos y Hahto (2012); Baker (2010); Nielsen (2010); Rubio y Giménez (2014) y Shurtzy von Isenburg (2011), en cuanto a que una vez usado el dispositivo gran parte de la gente se inclina a pensar que es igual que el impreso, pero otro grupo sigue prefiriendo el impreso.

Para finalizar, los resultados, aunque descriptivos, señalan que las actitudes hacia el Reader mejoraron después de la experiencia de uso. La mayoría de los estudiantes de la muestra no poseía Reader antes de realizar la experiencia, y al preguntarles su opinión sobre el Reader plantearon que no les interesaba o no se sentían cómodos con el dispositivo. Después de realizar la experiencia de uso, la opinión mayoritaria (un $83 \%$ ) fue que era un complemento del texto impreso, e incluso un quinto de la muestra consideró que era mejor que leer en pantalla. Estos resultados son similares a los hallados por otros investigadores después de las experiencias de uso (Baker, 2010; Connell et al., 2012; Lartigue et al., 2013; Nielsen, 2010). $\mathrm{Si}$ bien, los porcentajes totales se inclinan a favor del texto impreso, es posible que aumente la aceptación de los dispositivos digitales a medida que estos se vuelvan más conocidos y accesibles por los estudiantes.

En cuanto a las limitaciones de esta investigación, la primera se deriva de la muestra empleada. En primer lugar, se trata de un conjunto de voluntarios, todos alumnos de carreras relacionadas con la psicología. Este tema debería estudiarse en otras poblaciones. En segundo lugar, el tamaño de la muestra también presenta limitaciones a su generalización. Debería realizarse el estudio en una muestra de mayor tamaño. Respecto de los materiales, se podría trabajar con textos más largos o con otro tipo de texto. En cuanto a la evaluación de la comprensión, podría ser más exhaustiva y emplearse otras metodologías para evaluar la comprensión (ejemplo, resúmenes).

En conclusión, se podría afirmar que existen diferencias en la comprensión lectora de textos expositivos entre el soporte impreso y el Reader. Este efecto no es global, ya que depende de los otros factores estudiados, el conocimiento previo y la aptitud verbal. En conjunto, los resultados 
de este estudio se pueden resumir de la siguiente forma: con el Reader, las personas con alta aptitud verbal construyeron mejor Base de Texto; y el Reader colaboró en la comprensión profunda de textos difíciles. Esto parecería sugerir que el Reader presentaría un desafío en la lectura, que beneficiaría a los lectores competentes, y/o que podría promover mayor actividad metacognitiva en los procesos de comprensión, siendo estas cuestiones a abordar en futuras investigaciones.

Respecto de la autoevaluación del rendimiento lector los estudiantes mayoritariamente reconocen que fue el mismo en ambos soportes: impreso y Reader, le siguió el impreso, y en último lugar el Reader. Es posible que la autoevaluación refleje la dificultad en dominar al soporte. Cabría ampliar en futuras investigaciones los aspectos metacognitivos, incluyendo aspectos como actitudes frente a la tecnología, ansiedad en la tarea y similares. Las opiniones que los participantes manifestaron al inicio de la experiencia cambiaron después de la experiencia de uso del dispositivo Reader, en un sentido más favorable. Futuras investigaciones pueden relacionar el acceso a dispositivos similares con cambios en la eficacia en su uso, y el aprovechamiento en ámbitos escolares y académicos.

\section{Referencias}

Afflerbach, P., Pearson, P., \& Paris, S. (2007). Skills and strategies: Their differences, their relationships, and why it matters. En K. Mokhtari\& R. Sheorey. (Eds.). Reading strategies in first- and second-language learners: See how they read (pp. 11-24). Norwood, MA: Christopher Gordon.

Ahlroos, P. \& Hahto, J. (2012). The e-Reader-An educational or an entertainment tool?- E-Readers in an academic setting. Liber Quarterly: The Journal of European Research Libraries, 21, 249-261.

Albarello, F. (2011). Leer/navegar en Internet. Las formas de lectura en la computadora. Buenos Aires: La Crujía.

Alonso Arévalo, J., Cordón García, J., \& Gómez Díaz, R. (2011). El libro electrónico en la biblioteca universitaria y de investigación. Biblos, 42, 15-35.

Amadieu, F., van Gog, T., Paas, A., \& Mariné, C. (2009). Effects of prior knowledge and concept-map structure on disorientation cognitive load, and learnig. Learning and Instruction, 19, 376-386.

Baker, R.D. (2010). Comparing the readability of text displays on paper, e-book Readers, and small screen devices. Tesis Doctoral.Denton: University of North Texas.

Benéitez, L.M., Camarero, C., \& San José, R. (2012). Tecnologías útiles para el consumidor pero con riesgo para las industrias de contenidos. El caso del libro electrónico. Universia Business Review, 2,82-101.

Burin, D. I., Barreyro, J.P., Saux, G., \& Irrazabal, N. (2015). Navigation and comprehension of expository hypertext: Hypertext structure, previous domain knowledge, and working memory capacity. ElectronicJournal of Research in Educational Psychology, 13(3), 529-550.
Burin, D., Kahan, E., Irrazabal, N., \& Saux, G. (2010). Procesos cognitivos en la comprensión de hipertexto: papel de la estructura del hipertexto, de la memoria detrabajo, y del conocimiento previo. Actas Congreso Iberoamericano de Educación, 1-12.

Burin, D., Kahan, E., Irrazabal, N., \& Saux, G. (2014). Comprensión de contenidos científicos en formato hipertextual: La estructura de navegación tiene efectos distintos según el conocimiento previo y la capacidad de memoria de trabajo. En: V. Jaichenco (Comp.). Psicolingüística en español. Homenaje a Juan Seguí (pp. 345-355). Buenos Aires: Eudeba.

Campbell, A., Callaghan, G., McGarvie, D.W., \& Hynd, M. (2013). Do students study and learn differently using e-Readers? A cross-discipline research investigation into the pedagogical implications of using e-Readers to study university level texts.The QAA Enhancement Themes International Conference on Enhancement and Innovation in Higher Education. Glasgow, UK.

Connell, C., Bayliss, L., \& Farmer, W. (2012).Effects of eBook Readers and tablet computers on reading comprehension. International Journal of Instructional Media, 39(2), 131-140.

Cordón García, J.A., Alonso Arévalo, J. \& Martín Rodero, H. (2010). Los libros electrónicos: la tercera ola de la revolución digital. Anales de Documentación, 13, 53-80.

Cortada de Kohan, N. (2004). Baires. Test de Aptitud Verbal "Buenos Aires". Madrid: Tea.

Davis, D. \& Woody, W. (2013). E-textbooks at what cost? Performance and use of electronic vs. print texts. Computers \& Education, 62,18-23. doi: 10.1016/j. compedu.2012.10.016

Federación de Gremios de Editores (2011). Hábitos de lectura y compra de libros. Informe técnico. Madrid: Conecta.

Fernández Gómez, M. J., Cordón-García, J. A., AlonsoArévalo, J., \& Gómez-Díaz, R. (2013). Prácticas de consumo electrónico: los lectores ante los nuevos soportes. En: Cordón-García, J. A., Gómez-Díaz, R., \& Alonso-Arévalo, J. (2014). Documentos electrónicos y textualidades digitales: nuevos lectores, nuevas lecturas, nuevos géneros (pp. 177-219).Salamanca: Ediciones Universidad de Salamanca.

Gielen, N. (2010). Handheld e-book Readers and scholarship: Report and Reader survey. New York, NY: American Council of Learned Societies.

Graesser, A., McNamara, D., \& Van Lehn, K. (2005).Scaffolding deep comprehension strategies through point \& query, auto tutor, and iSTART. Educational Psychologist, 40(4), 225-234. doi: 10.1207/s15326985ep4004 4

Irrazabal, N., Burin, D., \& Saux, G. (2012).Conocimiento previo y memoria de trabajo en la comprensión de textos expositivos. Revista Argentina de Ciencias del Comportamiento, 4(2), 11-18.

Kintsch, W. (1996). El rol del conocimiento en la comprensión del discurso: un modelo de construcción-integración. En K. Goodman \& W. Kintsch. Textos en contexto 2. Los procesos de lectura y escritura (pp. 71-133). Buenos Aires: Kapeluz.

Lartigue, J., Rutledge, R., \& Rice, A. (2013). Paper or pixel: assessing reading performance across multiple mediums. En J. Herrington, A. Couros \& V. Irvine (Eds.).Proceedings of EdMedia: World Conference on Educational Media and Technology 2013 (pp. 1045 1052). Association for the Advancement of Computing in Education (AACE). 
León, J.A. \& Escudero, I. (2003).Protocolos verbales en el estudio de las inferencias: una metodología emergente. En León, J.A. (coord.). Conocimiento y discurso. Claves para inferir y comprender (pp.99-119). Madrid: Pirámide.

McNamara, D., Kintsch, E., Songer, N., \& Kintsch, W. (1996). Are good texts always better? Interactions of text coherence, background knowledge, and levels of understanding in learning from text. Cognition and Instruction, 14(1), 1-43. doi: 10.1207/s1532690xci1401_1

Margolin, S.J., Driscoll, C., Toland, M.J., \& Kegler, J.L. (2013). E-Readers, computer screens, or paper: Does reading comprehension change across media platforms? Applied Cognitive Psychology, 27, 512-519. doi: 10.1002/acp. 2930

Nielsen, J. (2010). iPad and Kindle Reading Speeds. Disponible en: http://www.useit.com/alertbox/ipad-kindlereading.html

Perfetti, C. (2007). Reading ability: Lexical quality to comprehension. Scientific Studies of Reading, 11(4), 357-383. doi: 10.1080/10888430701530730

Perfetti, C. \& Stafura, J. (2014).Word knowledge in a theory of reading comprehension. Scientific Studies of Reading, 18 (1), 22-37. doi: 10.1080/10888438.2013.827687

Rainie, L., Zickuhr, K., Purcell, K., Madden, M., \& Brenner, J. (2012). The rise of e-reading. Washington, D.C: Pew Research Center's Internet \& American Life Project.

Rich, S. (2012).The influence of electronic books on third grade reading comprehension. A Master's Project of Science in Education Literacy Education.Fredonia, New York: State University of New York at Fredonia. Department of Education.

Rockinson-Szapkiw, A. J., Courduff, J., Carter, K., \& Bennett, D. (2012).Electronic versus traditional print textbooks: A comparison study on the influence of university students' learning. Computers \& Education, 6, 259-266. doi: 10.1016/j.compedu.2012.11.022
Rockinson-Szapkiw, A. J., Wendt, J., \& Lunde, R. (2013). Electronic versus print textbooks: the influence of textbook format on university students' self-regulated learning strategies, motivation, and text anxiety. American Journal of Distance Education, 27(3), 179-188. doi: $10.1080 / 08923647.2013 .796230$

Rubio, G. A., \& Giménez, B. G. D. (2014). Papel vs. digital: hábitos de lectura de los estudiantes de la Universidad Europea de Madrid (UEM). Caracteres.net, 3(1), 241-271

Schugar, J.T., Schugar, H., \& Penny, C. (2011). A nook or a book? Comparing college students' reading comprehension levels, critical reading, and study skills. International Journal of Technology in Teaching and Learning, 7(2), 174-192.

Shibata, H. \& Omura, K. (2012).Comparing Paper Books and Electronic Media in Reading to Answer Questions. International Conference on Digital Printing Technologies, 1, 43-46.

Shurtz, S.\& von Isenburg, M. (2011).Exploring e-Readers to support clinical medical education: two case studies. Journal of the Medical Library Association,99(2), 110. doi: 10.3163/1536-5050.99.2.002.

Van Dijk, T. \& Kintsch, W. (1983).Strategies of discourse comprehension. Nueva York: Academic Press.

Wexelbaum, R., Miltenoff, P., \& Parault, S. (2011). EBooks and reading comprehension: Perspectives of librarians and educators. Bibliosphera, 14(3), 1-12.

Zhang, L. (2012). Changes in reading behavior among eReader adopters. (Tesis doctoral). Rochester Institute of Technology, New York, U.S.A. 